\title{
A Case Study - Investigation of Rounded Corners Effect on Corrugated Bulkheads
}

\author{
Özgür Özgüç \\ Department of Naval Architecture and Ocean Engineering, \\ Istanbul Technical University, \\ Maslak, Istanbul, Turkey, +90 2122856452 \\ ozguco@itu.edu.tr \\ Received: $30^{\text {th }}$ November 2016 \\ Accepted: 2nd January 2017 \\ DOI; 10.18466/cbayarfbe.319882
}

\begin{abstract}
Corrugated bulkheads are widely used in ship and offshore structures giving higher load carrying capacity compared to the flat plates. As a case study this paper attempts to investigate the effect of rounded corners on corrugated bulkheads with respect to buckling strength. Non-linear finite element analysis using ABAQUS is utilized. Rounded and sharp comers are being compared for a typical geometry with three different plate-thicknesses in order to find out if the rounded corners give additional strength and therefore can be used to reduce the flange-width. It is shown that the linear buckling load is larger for round cornered bulkheads. However, the ultimate strength is smaller than for a similar bulkhead with sharp comers. The results and insights derived from the present work are drawn.
\end{abstract}

Keywords - Axial compression, Bending, Buckling, Corrugated bulkheads, Non-linear finite element analysis, Ultimate limit state

\section{Introduction}

Corrugated panels have been commonly used for the structures in engineering field. There is also an increasing demand for the accommodation units fabricated of corrugated panels in ship and offshore facilities. The trapezoidal panels have been commonly used for decks, stiffeners, roofing, girders, wall cladding, within offshore facilities and sandwich panel structures and are easy, light to form and can give a higher load carrying capacity compared to flat panels. Generally, corrugations are rectangular, trapezoidal, triangular and sinusoidal. The trapezoidal and triangular sections can be considered as approximate forms of sinusoidal corrugations. Due to the corrugated profile, these plates have high inherent stiffness. Corrugated panels are self-stiffened and under various practical applications, the panel is exposed to sides loads, in plane forces, shearing and bending forces.

Corrugated plates are plates with inbuilt stiffness. Their higher depth due to the corrugations attribute to higher structural rigidity. Corrugated plates have higher stiffness to weight ratio and high strength to weight ratio. For a high performance structure, corrugated plates can be recommended because of the above special structural features and/properties and low fabrication cost. A typical corrugated bulkhead structure is shown for a tanker vessel under construction in Fig 1.

Two alternative designs with corrugated shell plate are used with regards to corrugation symmetry. The first one had unequal flanges and the same thickness as that of the reference stiffened model. The second had equal flange lengths. The weight reduction 
figures accomplished with using these designs were 38 percent and 59 percent in shell plate, respectively, as compared with a reference model for conventional stiffened shell plate. The work concluded with a reduction in production costs by 35 percent and 49 percent, respectively. The investigation clearly shows that corrugated panels are frequently favorable for lightweight design and fabricating robustness of ship and offshore structures [9].

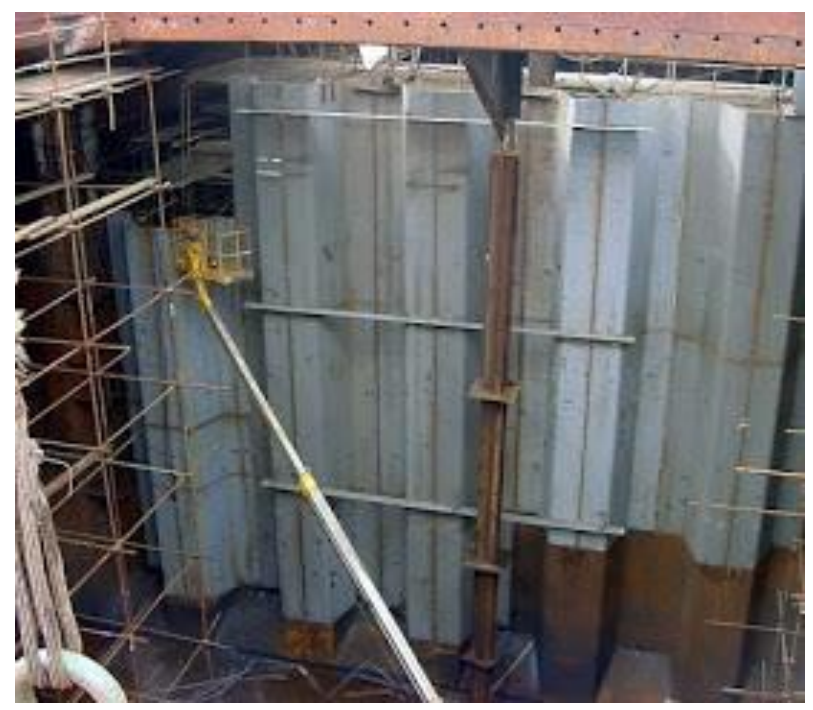

Figure 1. A typical corrugated bulkhead structure shown

Karthi and Nandakumar [4] performed the parametric investigation into the linear elastic behavior of corrugated plates with trapezoidal profile under variations in thickness, depth of corrugation and angle of corrugation, when they were subjected to various loadings with different boundary conditions. The stress analyses were done with the ANSYS finite element software.

Lei Et al. [6] carried out FEM analyses on the ultimate strength capacity of the corrugated bulkheads under linear hydrostatic pressure by means of ANSYS software, taking into account of geometry non-linearity and material non-linearity. Many influence factors would affect the ultimate strength of corrugated bulkheads, including corrugate angle, plate thickness, boundary condition and steel consumption, a discussion of different variables of the above influence is given. By the computational analyses, it showed that increasing either corrugate angle or plate thickness had effect on ultimate strength of corrugated bulkheads and unit consumption of steel.

Rinsberg Et al. [8] compared corrugated type plate with a traditional stiffened plate with regards to geometrical and operational features such as weight, strength, and cost. In this aim, an optimization approach was improved for ship and offshore units. The method enabled analysis and comparison of different resolutions with respect to strength properties, cost and weight. The structural strength properties cover buckling capacity, ultimate limit state, and fatigue damage assessments. Findings demonstrated as compared to conventional stiffened plate, corrugated type made by shell plate could be employed as a better competitive lightweight design resolution. That could be constructed and fitted with a lower cost.

Corrugated panels were also utilized as part of blast units on ship and offshore structures. Even though the numerical approaches in detail were frequently employed, the simplified approaches in accordance with using spring and mass idealization, for instance Biggs' formula, were playing an important role in initial design stage. The approach of a simplified theoretical was made and new transformation items for plates using straight end plates were obtained. This approach was verified with both experimental results and finite element analyses. An acceptable agreement was accomplished by Liang Et al. [7].

Sun and Spencer [9] presented accommodation units in ship and offshore facilities fabricated with corrugated plates so as to reduce the cost and fabrication timing. The discrepancy from corrugated bulkhead structures in offshore and ship facilities, corrugated plates used for the accommodation units are being designed with the types of trapezoidal or triangular section with non-uniform beams and having corrugation angle varying from 45 and 90 .

Ji Et. al [5] used simplified methods so as to determine the capacity of ultimate limit state (ULS) of corrugated type structures which were obtained with using the beam-column theory. The methods could consider of the effects of shear force and adjacent structures. It was clearly demonstrated that the methods obtained from the study were correct 
and reliable in the engineering aims. Furthermore, a stronger design software was also developed.

This present study attempts to investigate the effect of rounded corners on corrugated bulkheads with respect to buckling strength. Det Norske Veritas (DNV) ship rules [2, 3] states that corrugated bulkheads should be calculated with a flange breadth based on the intersection point between the middle lines for flange and web. This means that the radius should be disregarded while calculating buckling strength of the bulkhead.

Non-linear finite element analysis using ABAQUS [1] is utilized. Rounded and sharp comers are being compared for a typical geometry with three different plate-thicknesses. This is carried out to find out if the rounded corners give additional strength and therefore can be used to reduce the flange-width. The analyses consider local buckling of corrugated bulkheads with two different load conditions, pure axial load and pure bending.

It is drawn that the linear buckling load is larger for round cornered bulkheads. However, the ultimate strength is smaller than for a similar bulkhead with sharp comers. It is therefore not advised to reduce the flange breadth in the ship class rule formulations $[2,3]$.

\section{Background and Motivation}

Major Classification Society ship rules such as Det Norske Veritas (DNV) $[2,3]$ states that corrugated bulkheads should be calculated with a flange breadth based on the intersection point between the middle lines for flange and web. This means that the radius should be disregarded while calculating buckling strength of these bulkheads. In this regard, both rounded and sharp corners are being compared for a typical geometry with three different platethicknesses in order to find out if the rounded corners give additional strength and therefore can be used to reduce the flange-width in DNV ship rule formula.

Part of a corrugated bulkhead with rounded corners and sharp corners are illustrated in Fig 2 and Fig 3.

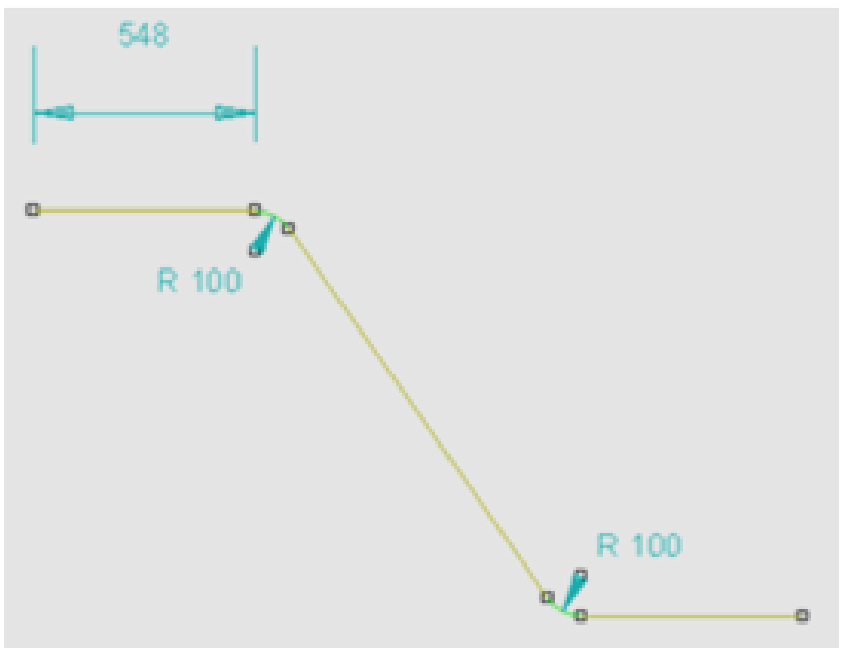

Figure 2. Part of a corrugated bulkhead with rounded corners

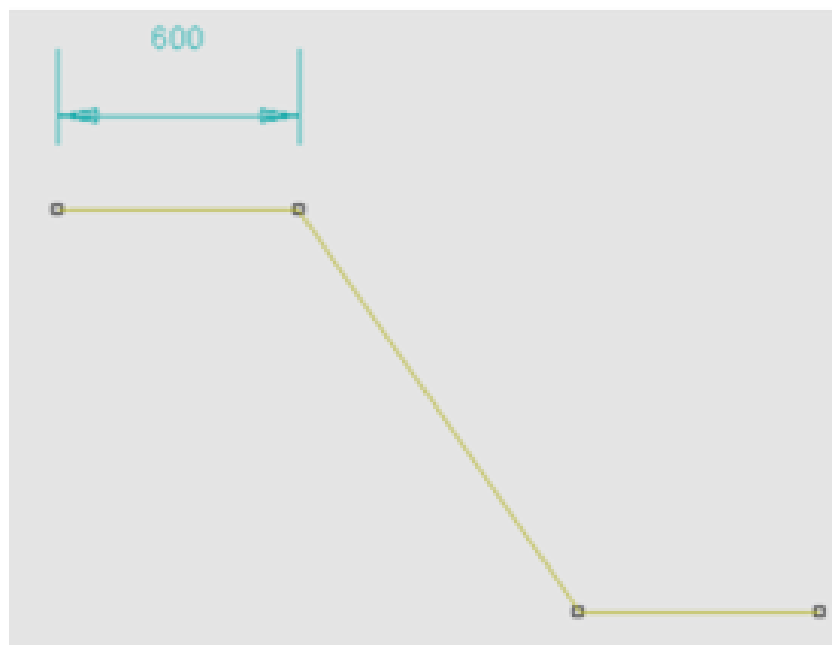

Figure 3. Part of a corrugated bulkhead with sharp corners

Non-linear finite element analysis by means of ABAQUS [1] tool is utilized in order to perform the structural investigations in this study.

\section{Non-linear Finite Element Analysis}

The geometry to be analyzed is shown in Fig 4. In all of the analysis the plate length is set to $4000 \mathrm{~mm}$. This is found to be sufficient to describe the behaviour of longer plates as well. The plate thickness is set to 10,20 and $25 \mathrm{~mm}$, where this covers different degrees of slenderness in typical ship design. 


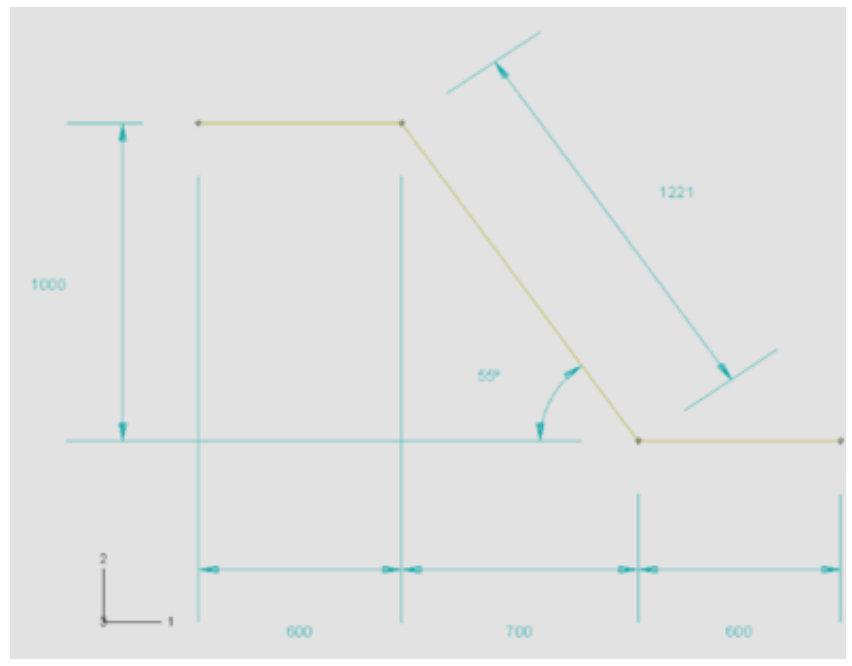

Figure 4. The geometry that has been analyzed

All of the analysis uses the material properties of the steel grade high tensile HT32 [2], where the main particulars are presented in Table 1. For material non-linearity a bi-linear material model is applied to account for hardening in the material after yielding.

Table 1. Material properties

\begin{tabular}{|l|l|}
\hline$\sigma_{f}$ & $315 \mathrm{MPa}$ \\
\hline$E$ & $208000 \mathrm{MPa}$ \\
\hline$E^{\top}$ & $1000 \mathrm{MPa}$ \\
\hline$v$ & 0.3 \\
\hline
\end{tabular}

For both load cases the external load is applied at one edge while the opposite is simply supported. All of the edges are forced to remain straight, but they are generally free to move in plane. This is performed so as to prevent stresses due to the fact that contraction of the material. On the longitudinal edges a symmetry-criterion is applied. Fig 5 and Fig 6 show the plates with boundary conditions.

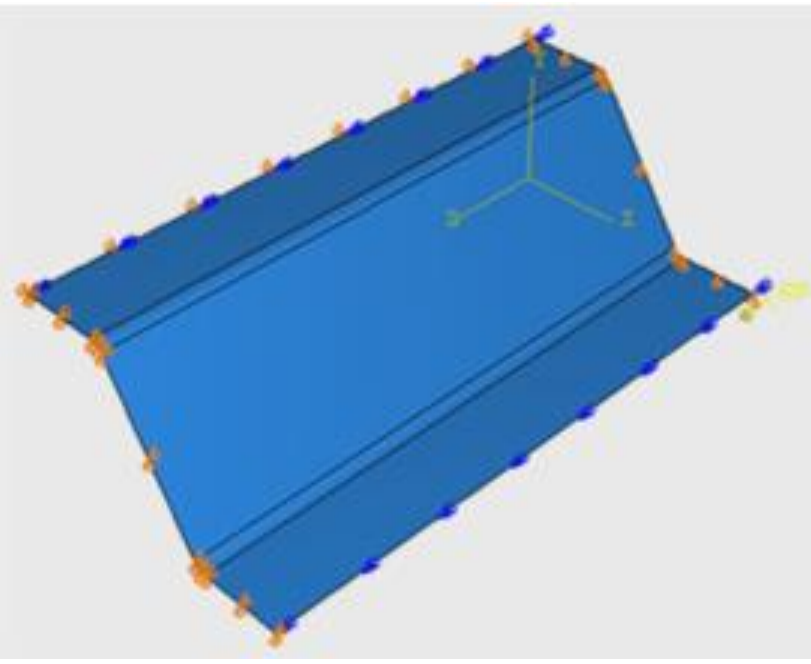

Figure 5. Boundary conditions for the plate exposed to axial compression

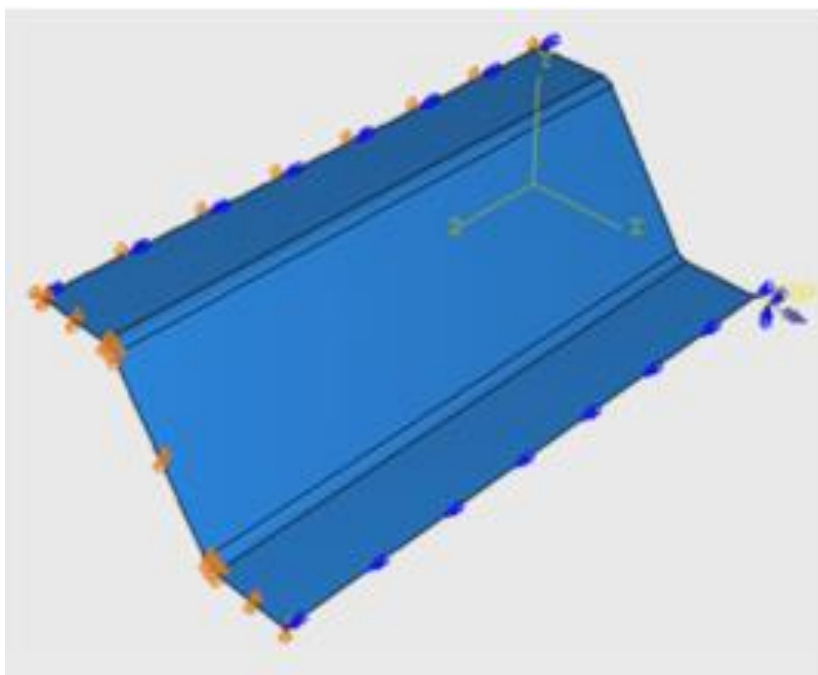

Figure 6. Boundary conditions for the plate exposed to bending

\section{Analysis Results}

In Fig 7 and Fig 8, the deformed shape of the bulkhead is shown when von Mises yield occurs in the middle plane of the plate.

The results of the analysis are presented in Table 2. Here it is seen that the linear buckling-load is slightly larger for bulkheads with rounded corners compared to similar bulkheads with sharp corners. However, the actual ultimate strength is lower. The results are qualitatively the same for both axially compressed and bent bulkheads. Due to numerical problems only one bending analysis is performed. 


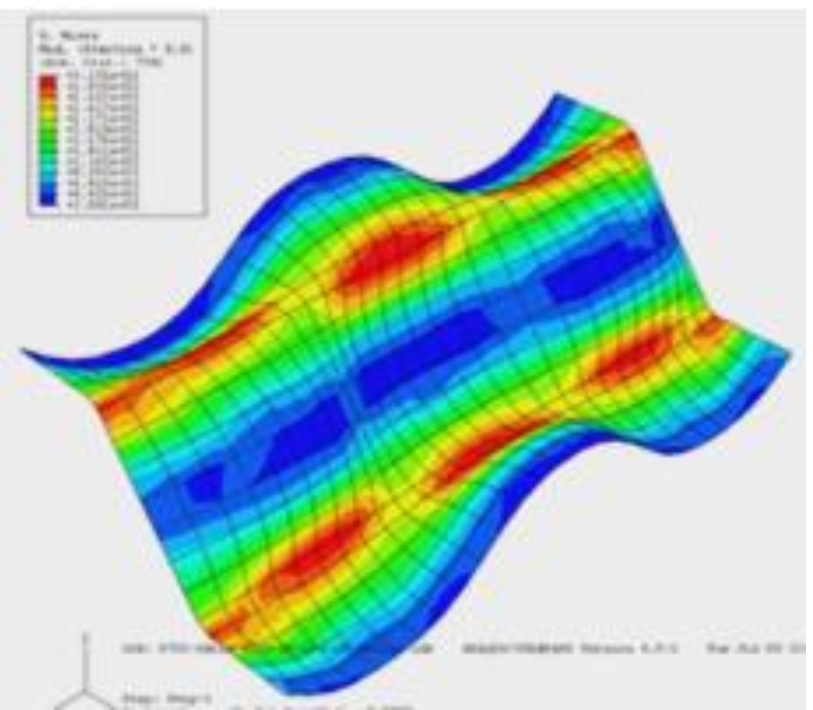

Figure 7. Stress-plot. $10 \mathrm{~mm}$ corrugated plate exposed to axial compression

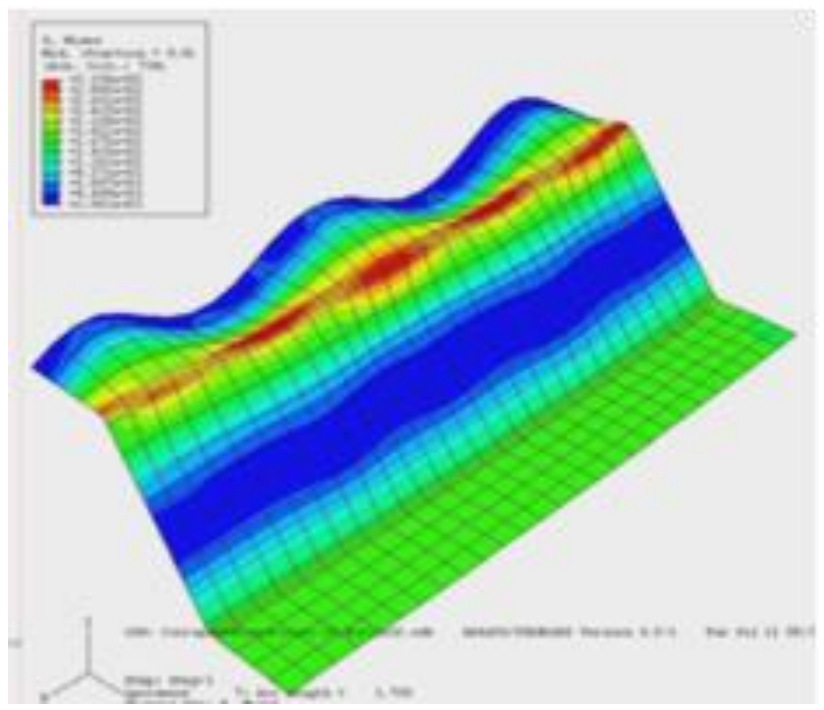

Figure 8. Stress-plot. 10mm corrugated plate exposed to pure bending

Table 2. A summary for the results of the non-linear analyses

\begin{tabular}{|c|c|c|c|c|c|c|c|c|}
\hline & \multirow{2}{*}{\multicolumn{2}{|c|}{$\begin{array}{c}\text { Bending* } \\
\text { tp }=10\end{array}$}} & \multicolumn{6}{|c|}{ Axial compression ${ }^{* *}$} \\
\hline & & & \multicolumn{2}{|c|}{$\mathrm{tp}=10$} & \multicolumn{2}{|c|}{$\mathrm{tp}=20$} & \multicolumn{2}{|c|}{$\mathrm{tp}=25$} \\
\hline & $\sigma_{\mathrm{el}}$ & $\sigma_{\text {uls }}$ & $\sigma_{\mathrm{el}}$ & $\sigma_{\text {uls }}$ & $\sigma_{\mathrm{el}}$ & $\sigma_{\text {uls }}$ & $\sigma_{\mathrm{el}}$ & $\sigma_{\text {uls }}$ \\
\hline$r=0$ & 51,21 & 121,33 & 53,17 & 130,26 & 212,05 & 206,82 & 330,68 & 247,70 \\
\hline$r=100$ & 51,60 & 116,73 & 58,61 & 126,65 & 215,50 & 202,94 & 332,48 & 243,77 \\
\hline $\begin{array}{c}\text { Ratio } \\
(r=100 / r=0)\end{array}$ & 1,01 & 0,96 & 1,10 & 0,97 & 1,02 & 0,98 & 1,01 & 0,98 \\
\hline
\end{tabular}

*Bending stress is calculated by $\sigma_{1}=M_{b} \cdot h_{w} / 2 \cdot I$

${ }^{*}$ Axial compression stress is calculated by $\sigma_{1}=P_{c} / A$

Fig 9 shows the relation between load and shortening for the $10 \mathrm{~mm}$ corrugated panel subjected to axial compression, and Fig 10 illustrates the relation between moment and curvature for the panel subjected to pure bending. The Stresses and strains are made dimensionless with respect to the yield stress and yield strain.

The panel with rounded corners subjected to bending has not passed its ultimate limit state. However, there is a good reason to believe that the maximum load is reached.

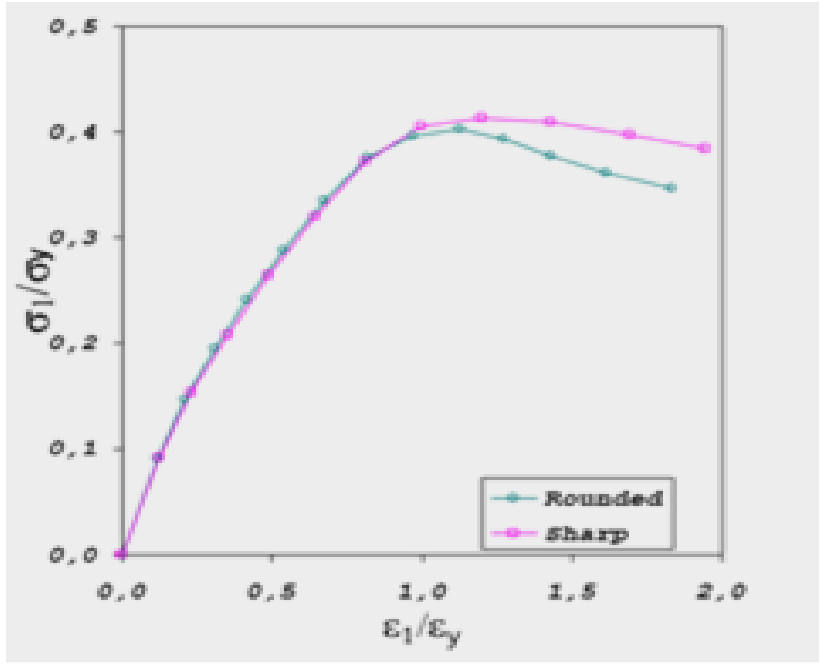

Figure 9. Load-shortening curve for $10 \mathrm{~mm}$ corrugated plate exposed to axial compression 


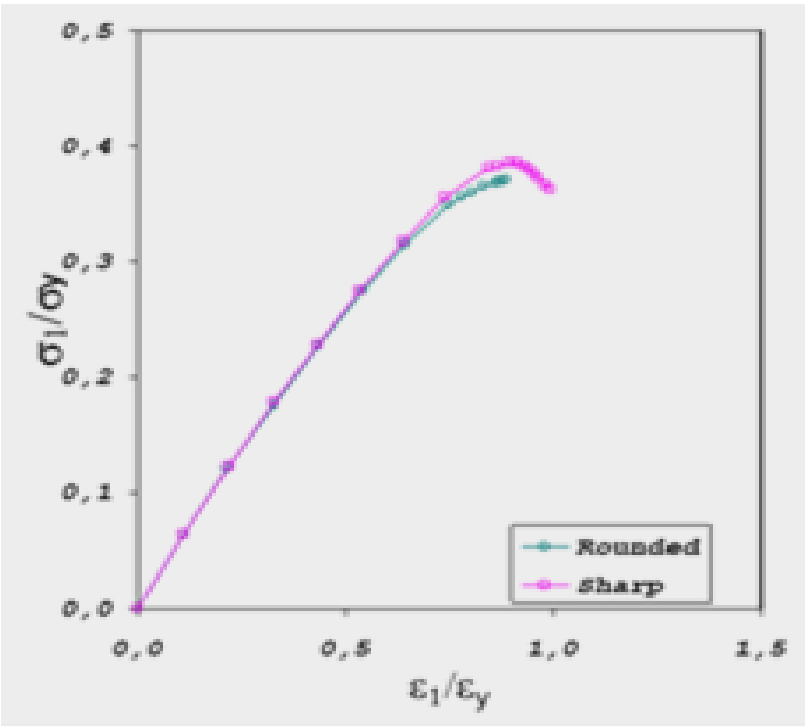

Figure 10. Moment-curvature curve for $10 \mathrm{~mm}$ corrugated plate exposed to pure bending

\section{Concluding Remarks}

Non-linear finite element analyses using ABAQUS are utilized to assess the difference in strength between rounded and sharp corners in corrugated panels. The analyses are being carried out for a typical geometry with three different platethicknesses. The analysis considers buckling of corrugated bulkheads under pure axial compression and pure bending. These results indicate that corrugated panels with rounded corners are weaker than similar panels with sharp corners. Thus it will be non-conservative to use a reduced flange breath in the Classification Rules such as DNV ship rule formulas for assessing the ultimate strength of corrugated panels with rounded corners. It is also draw that the difference in strength between rounded and sharp corners are rather small, i.e. in the range of $2-4 \%$ for the cases studied.

\section{References}

[1] ABAQUS, User's Manual, Hibbitt, Karlsson and Sorensen, 2009.

[2] Det Norske Veritas (DNV), Rules for Classification of Ship, Part 3, Chapter 1, Sec.9, January 2014.

[3] Det Norske Veritas (DNV), Rules for Classification of Ship, Part 2, Chapter 2, Sec.1, January 2015.
[4] Karthi, L.; Nandakumar, C.G. Investigations on elastic behaviour of corrugated plates, International Journal of Scientific \& Engineering Research, 2013; 4(5), 1-9.

[5] Ji, H.D.; Cui, W.C.; Zhang, S.K. Ultimate strength analysis of corrugated bulkheads considering influence of shear force and adjoining structures, Journal of Constructional Steel Research, 2001; 57(5), 525-545.

[6] Lei, H.; Ping, Y. Numerical analysis on the ultimate strength of corrugated bulkheads, Chinese Journal of Ship Research, 2011, 6(3), 28-31.

[7] Liang, Y.H.; Louca, L.A.; Hobbs, R.E. Corrugated panels under dynamic loads, International Journal of Impact Engineering, 2007, 34(7), 1185-1201.

[8] Ringsberg, J.; Sağlam, H.; Sarder, M.A.; Ulfvarson, A. Optimization of corrugated shell plating for marine structures, 2011, Proceedings of the ASME Thirtieth International Conference on Ocean, Offshore and Arctic Engineering (OMAE2011-50216), 1: 769-778.

[9] Sun, H.H.; Spencer, J. Buckling strength assessment of corrugated panels in offshore structures, Marine Structures, 2005; 18(7-8) 548-565.

\section{Notation}

$\sigma_{1} \quad$ - $\quad$ Nominal uniform flange stress in longitudinal direction, unit $\mathrm{N} / \mathrm{mm}^{2}$

$\sigma_{f} \quad$ - $\quad$ Characteristic yield stress, unit

$\mathrm{N} / \mathrm{mm}^{2}$

$\sigma_{\mathrm{el}} \quad$ - $\quad$ Elastic compressive buckling stress,

unit $\mathrm{N} / \mathrm{mm}^{2}$

$\sigma_{\text {uls }} \quad$ - Maximum capacity stress of bulkhead, unit $\mathrm{N} / \mathrm{mm}^{2}$

$v \quad$ - Poisson's ratio

$\mathrm{s} \quad$ - $\quad$ Flange breadth, unit $\mathrm{mm}$

$t_{p} \quad-\quad$ Plate thickness, unit mm

$\mathrm{h}_{\mathrm{w}} \quad$ - Web height, unit mm

A $\quad$ - Cross section area, unit $\mathrm{mm} 2$

E - Young's modulus, unit N/mm2

$\mathrm{E}^{\top} \quad$ - $\quad$ Tangential modulus, unit N/mm2

I $\quad$ - $\quad$ Moment of inertia, unit $\mathrm{mm} 4$

$\mathrm{M}_{\mathrm{b}} \quad$ - $\quad$ Bending moment, unit Nmm

$\mathrm{P}_{\mathrm{c}} \quad-\quad$ Compression force, unit $\mathrm{N}$ 